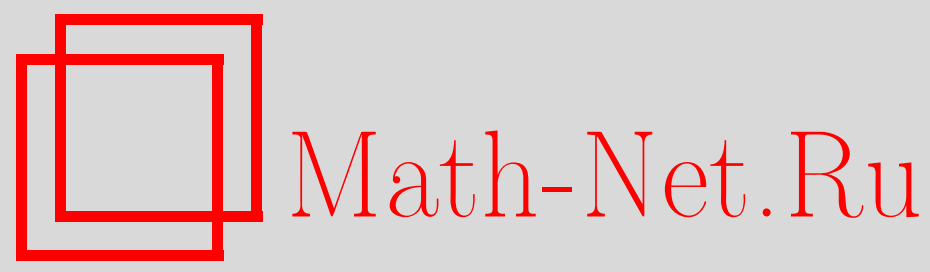

Г. И. Андриянов, Модифицированная проблема моментов в комплексной области, Матем. заметки, 2002, том 72, выпуск $6,804-814$

DOI: https://doi.org/10.4213/mzm467

Использование Общероссийского математического портала Math-Net.Ru подразумевает, что вы прочитали и согласны с пользовательским соглашением http://www . mathnet.ru/rus/agreement

Параметры загрузки:

IP: 54.237 .59 .107

26 апреля 2023 г., $17: 57: 17$

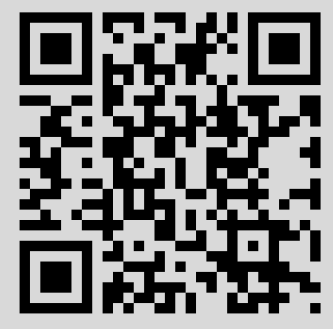


УДК 517.442

\title{
МОДИФИЦИРОВАННАЯ ПРОБЛЕМА МОМЕНТОВ В КОМПЛЕКСНОЙ ОБЛАСТИ
}

\section{Г.И. Андриянов}

\begin{abstract}
В работе рассмотрена проблема моментов в комплексной области. Полученные результаты обобщают и усиливают известный результат Казьмина. Указаны приложения полученных результатов в задаче Абеля-Гончарова (проблема единственности и конструкции), а также к полноте некоторых систем аналитических функций.

Библиография: 12 названий.
\end{abstract}

Пусть $D$ - односвязная ограниченная область с односвязньп дополнением $C D(\overline{\mathbb{C}} \backslash D)$ до всей расширенной комплексной плоскости $\overline{\mathbb{C}}_{z} ; A(D)$ - пространство функций $f(z)$, регулярньх в области $D$, с топологией равномерной сходимости на компактах из $D$.

Через $A_{0}(C D)$ обозначаем пространство функций $g(z), g(\infty)=0$, регулярных на $C D$, с топологией, индуцированной топологией сопряженного пространства, которое может быть реализовано в виде пространства $A(D)$.

Символом $A(\exp , D)$ обозначаем пространство целых функций $F(z)$, представимых в виде

$$
F(z)=\frac{1}{2 \pi i} \int_{\Gamma_{t}} e^{t z} g(t) d t,
$$

где $g(z) \in A_{0}(C D), g(z)$ - функция, ассоциированная по Борелю с $F(z), \Gamma_{t}-$ любой замкнутый жордановьй спрямляемый контур такой, что $g(z)$ регулярна на $\Gamma_{t} \cup \operatorname{ext} \Gamma_{t}$. Через int $\Gamma$, ext $Г$ обозначаем “внутреннюю" и “внешнюю" открытые области, на которые контур $\Gamma$ разбивает плоскость $\overline{\mathbb{C}}_{z}$. Таким образом,

$$
\overline{\mathbb{C}_{z}}=\operatorname{int} \Gamma \cup \Gamma \cup \operatorname{ext} \Gamma, \quad \operatorname{int} \Gamma \cap \operatorname{ext} \Gamma=\varnothing, \quad \infty \in \operatorname{ext} \Gamma .
$$

Хорошо известно, что пространство $A(\exp , D)$ изоморфно пространству $A_{0}(C D)$ (см. [1], [2]).

Можно считать, что топология пространства $A(\exp , D)$ "наводится" топологией пространства $A_{0}(C D)$, т.е. $F_{n}$ сходятся к $F(z)$ в пространстве $A(\exp , D)$ тогда и только тогда, когда функции $\gamma_{n}$, ассоциированные по Борелю с $F_{n}(z), n=0,1,2, \ldots$, сходятся в пространстве $A_{0}(C D)$ к функции $\gamma(z)$, ассоциированной по Борелю с $F(z)$ (см. [1], [2]).

Пусть имеется система функций $\left\{f_{n}(z)\right\}_{n=0}^{\infty}$, регулярных в области $D$. Хорошо известно следующее 
ОПРЕДЕЛЕниЕ 1. Система функций $\left\{f_{n}(z)\right\}_{n=0}^{\infty}$ называется полной в пространстве $A(D)$, если замыкание линейной оболочки данной системы (по топологии пространства $A(D))$ совпадает со всем $A(D)$.

В 70-х годах Казьмин рассмотрел следующую задачу [3].

Пусть имеется функция $W(z) \in A(D)$, которая является однолистной в области $D$. Помимо этого задаются $p, p \geqslant 1, p \in \mathbb{N}$, аналитических в области $D$ функций $A_{s}(z)$, $A_{s}(z) \not \equiv 0$, и $p$ последовательностей комплексных чисел $\left\{a_{n s}\right\}_{n=0}^{\infty}, s=0,1,2, \ldots, p-1$. Наконец, фиксируется произвольным образом набор из $p$ целых чисел $l_{s}, 0 \leqslant l_{s} \leqslant p-1$.

I. Спрашивается, существует ли функция $g(z) \in A_{0}(C D)$, для которой имеют место равенства

$$
\frac{1}{2 \pi i} \int_{\Gamma}[W(z)]^{n p+l_{s}} A_{s}(z) g(z) d z=a_{n s}, \quad n=0,1, \ldots, \quad s=0,1,2, \ldots, p-1
$$

где $\Gamma$ - замкнутьй жорданов спрямляемьй контур такой, что $g(z)$ регулярна на $\Gamma \cup \operatorname{ext} \Gamma$, $\Gamma \subset D$.

II. Предположим, что числовая совокупность $\bigcup_{s=0}^{p-1}\left\{a_{n s}\right\}_{n=0}^{\infty}$ допустима, т.е. сушествует функция $g(z) \in A_{0}(C D)$, для которой вьполняются соотношения (2).

Спрашивается, как по числам $\bigcup_{s=0}^{p-1}\left\{a_{n s}\right\}_{n=0}^{\infty}$ (моментам) из правой части (2) восстановить функцию $g(z) \in A_{0}(C D)$ ?

Решение задач I и II было названо общей проблемой моментов в комплексной области. Заметим, что к решению общей проблемы моментов приводят многие задачи по теории интерполяции в пространстве $A(\exp , D)[3] . \mathrm{K}$ подобному же кругу вопросов приводят и задачи о полноте в пространстве $A(D)$ некоторых систем аналитических в области $D$ функций. Заметим, что решение обшей проблемы моментов было получено Казьминым при условии, что образ области $D$ при отображении функцией $W(z)$ из (2) инвариантен относительно поворота на угол $2 \pi / p$ вокруг начала координат (область $G=W(D)$, обладающая этим свойством, назьвается $(2 \pi / p)$-инвариантной [3], [4]). Попытки снять условие $(2 \pi / p)$-инвариантности области $G$ успеха не имели [3], [4].

Данная работа посвящена решению следующей задачи. Пусть имеется $p, p \in \mathbb{N}$, $p \geqslant 2$, функций $W_{j}(z)$, которые голоморфны и однолистны в области $D$. Помимо этого задаются $p$ аналитических в $D$ функций $A_{j}(z), j=0,1,2, \ldots, p-1$, не имеющих нулей в области $D$ (это условие носит технический характер, см. ниже замечание 4 ), и $p$ последовательностей комплексных чисел $\left\{a_{n j}\right\}_{n=0}^{\infty}, j=0,1,2, \ldots, p-1$.

Спрашивается, существует ли функция $g(z) \in A_{0}(C D)$, для которой имеют место равенства

$$
\frac{1}{2 \pi i} \int_{\Gamma_{z}}\left[W_{j}(z)\right]^{n p+l_{j}} A_{j}(z) g(z) d z=a_{n j}
$$

$j=0,1,2, \ldots, p-1,0 \leqslant l_{j} \leqslant p-1, l_{j} \in \mathbb{N} \cup\{0\}, n=0,1,2, \ldots$ Здесь $\Gamma_{z}-$ любой замкнутый жордановьй спрямляемый контур такой, что $g(z)$ регулярна на $\Gamma_{z} \cup \operatorname{ext} \Gamma_{z}, \Gamma_{z} \subset D$, $\Gamma_{z}$ достаточно близок к границе области $D$.

Кроме того, возникает вопрос, как по числовой совокупности $\bigcup_{j=0}^{p-1}\left\{a_{n j}\right\}_{n=0}^{\infty}$ восстановить функцию $g(z)$ в $(3)$ ?

Ниже будем считать, что функции $\left\{W_{j}(z)\right\}_{j=0}^{p-1}$ в $(3)$ в общем случае различны, а образы области $D$ при отображениях функциями $W_{j}(z), j=0,1,2, \ldots, p-1$, не являются $(2 \pi / p)$-инвариантными. Задачу поиска функции $g(z)$ и условий допустимости для 
$\bigcup_{j=0}^{p-1}\left\{a_{n j}\right\}_{n=0}^{\infty}$ в $(3)$ назовем модифицированной проблемой моментов (М-проблемой моментов).

Очевидно, что М-проблема моментов содержит общую проблему моментов Казьмина при оговоренных вьше условиях на функции $A_{j}(z)$ в качестве весьма частного случая, а именно, в (3)

$$
W_{0}(z) \equiv W_{1}(z) \equiv W_{2}(z) \equiv \cdots \equiv W_{p-1}(z) \equiv W(z)
$$

и образ области $D$ при отображении функцией $W(z)$ является $(2 \pi / p)$-инвариантным множеством.

Данная работа состоит из двух основных частей. В первой части находится решение М-проблемы моментов. Во второй части указаны простейшие (но не тривиальные) приложения М-проблемы моментов к интерполяционной задаче Абеля-Гончарова и вопросам полноты некоторых систем аналитических функций в пространстве $A(D)$.

\section{1. Решение М-проблемы моментов}

Для того чтобы сформулировать основной результат, связанный с М-проблемой моментов, нам потребуются некоторые предположения о числовой совокупности $\bigcup_{j=0}^{p-1}\left\{a_{n j}\right\}_{n=0}^{\infty}$ в правой части равенств (3).

Будем считать, что числовая совокупность $\bigcup_{j=0}^{p-1}\left\{a_{n j}\right\}_{n=0}^{\infty}$ обладает следуюшим свойством:

$$
g_{j}(w) \equiv \sum_{n=0}^{\infty} \frac{a_{n j}}{w^{n p+l_{j}+1}} \in A_{0}\left(C G_{j}\right),
$$

$j=0,1,2, \ldots, p-1, p \in \mathbb{N}, p \geqslant 2$, где $G_{j}=W_{j}(D)$ - образы области $D$ при отображении однолистньми голоморфными функциями $W_{j}(z)$ из $(3)$.

ТЕОрема 1. Пусть $D$ - любая односвязная ограниченная область с односвязным дополнением $C D$ до $\overline{\mathbb{C}}_{z}$ и числовая совокупность $\bigcup_{j=0}^{p-1}\left\{a_{n j}\right\}_{n=0}^{\infty}$ удовлетворяет условию (5).

Решение М-проблемы моментов (3) в пространстве $A_{0}(C D)$ существует тогда и только тогда, когда существует совокупность из $p$ функиий $\left\{f_{j}(w)\right\}_{j=0}^{p-1}$, $p \in \mathbb{N}, p \geqslant 2$, обладающих следующими свойствами (А-условия):

1) $f_{j}(w) \in A_{0}\left(C G_{j}\right), f_{j}(w) \not \equiv 0, j=0,1,2, \ldots, p-1$;

2) $f_{j}(w)$ не имеют степеней вида $1 / w^{n p+l_{j}+1}$ в своем лорановском разложении в окрестности бесконечно удаленной точки, $j=0,1,2, \ldots, p-1$;

3) система функиий $\left\{f_{j}(w)\right\}_{j=0}^{p-1}$ удовлетворяет условию

$$
\begin{gathered}
\left\{g_{0}\left(W_{0}(z)\right)+f_{0}\left(W_{0}(z)\right)\right\} W_{0}^{\prime}(z) A_{j}(z)-\left\{g_{j}\left(W_{j}(z)\right)+f_{j}\left(W_{j}(z)\right)\right\} W_{j}^{\prime}(z) A_{0}(z) \in A(D), \\
j=1,2, \ldots, p-1 .
\end{gathered}
$$

Необходимость. Пусть М-проблема моментов (3) имеет решение $\gamma(z) \in A_{0}(C D)$, $\gamma(z) \not \equiv 0$. В таком случае, учитьвая однолистность функций $W_{j}(z), j=0,1,2, \ldots, p-1$, можем переписать (3) в эквивалентном виде:

$$
\frac{1}{2 \pi i} \int_{\Gamma_{j}} w^{n p+l_{j}} A_{j}\left(Z_{j}(w)\right) \gamma\left(Z_{j}(w)\right) Z_{j}^{\prime}(w) d w=a_{n j}, \quad n=0,1, \ldots
$$


где $\Gamma_{j}=W_{j}\left(\Gamma_{z}\right), \Gamma_{j} \subset G_{j}$, а контур $\Gamma_{z}$ описан в (3). Здесь $z=Z_{j}(w)$ - функции, обратные к $w=W_{j}(z)$ соответственно.

Из (5), (6) получаем

$$
\frac{1}{2 \pi i} \int_{\Gamma_{j}} w^{n p+l_{j}}\left\{A_{j}\left(Z_{j}(w)\right) \gamma\left(Z_{j}(w)\right) Z_{j}^{\prime}(w)-g_{j}(w)\right\} d w=0,
$$

$j=0,1,2, \ldots, p-1, n=0,1, \ldots$.

Представим функции $A_{j}\left(Z_{j}(w)\right) \gamma\left(Z_{j}(w)\right) Z_{j}^{\prime}(w)-g_{j}(w)$, каждую на соответствующем контуре $\Gamma_{j}$, в виде

$$
A_{j}\left(Z_{j}(w)\right) \gamma\left(Z_{j}(w)\right) Z_{j}^{\prime}(w)-g_{j}(w)=\Phi_{j}^{+}(w)+\Phi_{j}^{-}(w),
$$

$j=0,1,2, \ldots, p-1$, здесь $\Phi_{j}^{+}(w) \in A\left(\Gamma_{j} \cup \operatorname{int} \Gamma_{j}\right), \Phi_{j}^{-}(w) \in A_{0}\left(\overline{\mathbb{C}} \backslash \operatorname{int} \Gamma_{j}\right), \Phi_{j}^{-}(w) \not \equiv 0$ (см. [5]).

Подставляя (8) в (7), видим, что справедливо

$$
\frac{1}{2 \pi i} \int_{\Gamma_{j}} w^{n p+l_{j}} \Phi_{j}^{-}(w) d w=0, \quad j=0,1,2, \ldots, p-1, \quad n=0,1, \ldots
$$

Из $(8),(9)$ заключаем, что функции $\left\{\Phi_{j}^{-}(w)\right\}_{j=0}^{p-1}$ обладают свойствами 1$\left.), 2\right) A$-условий. Таким образом, остается проверить вьполнение свойства 3$) A$-условий.

Перепишем (в слегка измененной форме) представления (8) на контур $\Gamma_{z} \subset D\left(\Gamma_{j}=\right.$ $\left.W_{j}\left(\Gamma_{z}\right)\right)$ :

$$
\gamma(z)-g_{j}\left(W_{j}(z)\right) \frac{W_{j}^{\prime}(z)}{A_{j}(z)}=\widehat{\Phi}_{j}^{+}(z)+\Phi_{j}^{-}\left(W_{j}(z)\right) \frac{W_{j}^{\prime}(z)}{A_{j}(z)},
$$

здесь $\widehat{\Phi}_{j}^{+}(z) \in A\left(\Gamma_{z} \cup \operatorname{int} \Gamma_{z}\right), j=0,1,2, \ldots, p-1$.

Вычитая в (10) из первой строки $(j=0)$ вторую $(j=1)$ и вьполняя элементарные преобразования, получим краевое условие на контуре $\Gamma_{z}$ :

$\left\{\Phi_{0}^{-}\left(W_{0}(z)\right)+g_{0}\left(W_{0}(z)\right)\right\} W_{0}^{\prime}(z) A_{1}(z)-\left\{g_{1}\left(W_{1}(z)\right)+\Phi_{1}^{-}\left(W_{1}(z)\right)\right\} W_{1}^{\prime}(z) A_{0}(z)=\Psi^{+}(z)$, где $\Psi^{+}(z) \in A\left(\Gamma_{z} \cup \operatorname{int} \Gamma_{z}\right)$.

Повторяя подобные преобразования с первой $(j=0)$ и третьей $(j=2)$ строками в $(10)$ и т.д., заключаем, что для системы функций $\left\{\Phi_{j}^{-}(w)\right\}_{j=0}^{p-1}$ в $(8)$ вьполнены все пункты $A$-условий.

Достаточность. Пусть существует система функций $\left\{f_{j}(w)\right\}_{j=0}^{p-1}$, удовлетворяющая $A$-условиям. Образуем функции

$$
\Psi_{j}(w) \equiv g_{j}(w)+f_{j}(w), \quad j=0,1,2, \ldots, p-1,
$$

где $\left\{g_{j}(w)\right\}_{j=0}^{p-1}-$ функции, определенные в (5). Рассмотрим $p$ краевых задач Римана на контуре $\Gamma_{z} \subset D, \Gamma_{z}$ описан в (3). Нужно найти пары функций $\Phi_{j}^{-}(z) \in A_{0}\left(\overline{\mathbb{C}} \backslash \operatorname{int} \Gamma_{z}\right)$, $\Phi_{j}^{+}(z) \in A(D)$, связанных на контуре $\Gamma_{z}$ условием

$$
\frac{\Phi_{j}^{-}(z) A_{j}(z)}{W_{j}^{\prime}(z)}=\Phi_{j}^{+}(z)+\Psi_{j}\left(W_{j}(z)\right), \quad j=0,1,2, \ldots, p-1 .
$$


Каждая из краевых задач в (12) разрешима, и сушествует функция $\Phi_{j}^{-}(z) \not \equiv 0$, удовлетворяющая соответствующему краевому условию (см. [5]). Нетрудно проверить справедливость следующих равенств:

$$
\frac{1}{2 \pi i} \int_{\Gamma_{z}} W_{j}(z)^{n p+l_{j}} A_{j}(z) \Phi_{j}^{-}(z) d z=a_{n j}, \quad j=0,1,2, \ldots, p-1, \quad n=0,1, \ldots
$$

Покажем, что

$$
\Phi_{0}^{-}(z) \equiv \Phi_{1}^{-}(z) \equiv \Phi_{2}^{-}(z) \equiv \cdots \equiv \Phi_{p-1}^{-}(z), \quad z \in \Gamma_{z} \cup \operatorname{ext} \Gamma_{z} .
$$

Действительно, из (12) следуют соотношения

$$
\Phi_{j}^{-}(z)=\widehat{\Phi}^{+}(z)+\frac{\Psi_{j}\left(W_{j}(z)\right) W_{j}^{\prime}(z)}{A_{j}(z)}, \quad j=0,1,2, \ldots, p-1 .
$$

Вычтем из первой строки в (15) вторую и получим

$$
\Phi_{0}^{-}(z)-\Phi_{1}^{-}(z)=\widetilde{\Phi}^{+}(z)+\frac{\Psi_{0}\left(W_{0}(z)\right) W_{0}^{\prime}(z)}{A_{0}(z)}-\frac{\Psi_{1}\left(W_{1}(z)\right) W_{1}^{\prime}(z)}{A_{1}(z)},
$$

откуда, учитьвая (11) и $A$-условия, заключаем, что на $\Gamma_{z} \subset D$ справедливо

$$
\Phi_{0}^{-}(z)-\Phi_{1}^{-}(z)=\check{\Phi}^{+}(z),
$$

где $\check{\Phi}^{+}(z) \in A\left(\Gamma_{z} \cup \operatorname{int} \Gamma_{z}\right)$. Применяя в (16) теорему Лиувилля (см. [5]) и учитьвая равенство $\Phi_{0}^{-}(\infty)=\Phi_{1}^{-}(\infty)=0$, видим, что $\Phi_{0}^{-}(z) \equiv \Phi_{1}^{-}(z)$ в $C D$. Повторяя подобные рассуждения с первой и третьей строкой в (15) и т.д., получим (14). Из (13) и (14) следует, что М-проблема моментов разрешима. Решение М-проблемы моментов (это функция $\left.\Phi_{0}^{-}(z)\right)$ находится как решение задачи Римана в $(12)$ при $j=0$. Оно может быть записано в виде

$$
\Phi_{0}^{-}(z)=\frac{1}{2 \pi i} \int_{\Gamma_{t}} \frac{g_{0}\left(W_{0}(t)\right)+f_{0}\left(W_{0}(t)\right)}{A_{0}(t)(t-z)} W_{0}^{\prime}(t) d t, \quad z \in \operatorname{ext} \Gamma_{t} .
$$

Теорема доказана.

ЗАмечАниЕ 1. Теорема 1 сводит М-проблему моментов (3) к поиску системы функций $\left\{f_{j}(w)\right\}_{j=0}^{p-1}$, удовлетворяющей $A$-условиям.

ЗАмечАнИЕ 2 . В теореме 1 области $G_{j}=W_{j}(D), j=0,1,2, \ldots, p-1$, не обязаны быть $(2 \pi / p)$-инвариантньми, а функции системы $\left\{W_{j}(z)\right\}_{j=0}^{p-1}$ в общем случае различны между собой.

ЗАмечАниЕ 3. Теорема 1 дает вид решения М-проблемы моментов, которое описывается контурным интегралом (17).

ЗАмечание 4. Решение М-проблемы моментов получено в предположении, что функции $\left\{A_{j}(z)\right\}_{j=0}^{p-1}$ не имеют нулей в области $D$. Это ограничение не является принципиальньм, и решение М-проблемы моментов может быть получено для $A_{j}(z)$, имеющих конечное число нулей в области $D$. Однако в этом случае доказательство и формулировка теоремы 1 усложняются. В данной работе мы этого делать не будем.

ЗАмЕЧАнИЕ 5. В соответствии с теоремой 1 для того чтобы научиться находить решение М-проблемы моментов, достаточно научиться находить хотя бы $f_{0}(w)$ из системы функций $\left\{f_{j}(w)\right\}_{j=0}^{p-1}$, удовлетворяющей $A$-условиям. Ниже для некоторых случаев будет указан вполне конструктивный метод нахождения системы функций $\left\{f_{j}(w)\right\}_{j=0}^{p-1}$. 


\section{2. Приложения теоремы 1}

Рассмотрим интерполяционную задачу Абеля-Гончарова $(\mathrm{A}-\Gamma)$ : найти целую функцию $F(z)$ из некоторого пространства $A(\exp , D)$, удовлетворяющую условиям

$$
F^{(n)}\left(\lambda_{n}\right)=a_{n}, \quad n=0,1,2, \ldots,
$$

где числовая последовательность $\left\{\lambda_{n}\right\}_{n=0}^{\infty}-$ последовательность узлов интерполящии имеет следуюший вид:

$$
\lambda_{n}=n+b(n), \quad n=0,1, \ldots,
$$

здесь

$$
\left\{\begin{array}{l}
b(n)=b_{0} \text { при } n=2 k, \\
b(n)=b_{1} \quad \text { при } n=2 k+1, k=0,1,2, \ldots, \quad b_{0} \neq b_{1}, \quad b_{0}, b_{1} \in \mathbb{C} .
\end{array}\right.
$$

Покажем, как с помощью теоремы 1 решаются проблемы единственности и конструкции в задаче А-Г (18), (19) (относительно проблем единственности и конструкции (см. $[1],[2],[6]-[9])$.

Обозначим через $U$ следующую область:

$$
U=\left\{z=\rho e^{i \varphi}: 0 \leqslant \rho<\frac{\pi-|\varphi|}{\sin |\varphi|},|\varphi| \leqslant \pi\right\} .
$$

Область $U$ впервые была введена Гончаровым и Гельфондом (см. [7], [8]). Хорошо известно, что функция $w=z e^{z}$ голоморфна и однолистна в области $U$ и отображает ее на плоскость $\mathbb{C}_{w}$ с разрезом от точки $u=-1 / e(w=u+i v)$ вдоль отрицательной части действительной оси и до бесконечности (см. [9]).

2.1. Решение задачи Абеля-Гончарова. Пусть $D$ - любая фиксированная односвязная ограниченная область с односвязным дополнением: $\bar{D} \subset U(\bar{D}$-замыкание области $D)$. Представим числовую последовательность $\left\{a_{n}\right\}_{n=0}^{\infty}$ в правой части $(18)$ в виде

$$
\left\{a_{n}\right\}_{n=0}^{\infty}=\left\{a_{2 n}\right\}_{n=0}^{\infty} \cup\left\{a_{2 n+1}\right\}_{n=0}^{\infty} .
$$

Потребуем, чтобы числовые последовательности $\left\{a_{2 n}\right\}_{n=0}^{\infty}$ и $\left\{a_{2 n+1}\right\}_{n=0}^{\infty}$ обладали следующим свойством:

$$
\left\{\begin{array}{l}
g_{0}(w) \equiv \sum_{n=0}^{\infty} \frac{a_{2 n}}{w^{2 n+1}} \in A_{0}(C D) \\
g_{1}(w) \equiv \sum_{n=0}^{\infty} \frac{a_{2 n+1}}{w^{2 n+2}} \in A_{0}(C D)
\end{array}\right.
$$

где $G=W(D), w=z e^{z}$.

ТЕОРема 2. Пусть $D$ - любая односвязная ограниченная область с односвязным дополнением $C D$ до $\overline{\mathbb{C}_{z}}, \bar{D} \subset U$ и числовая последовательность $\left\{a_{n}\right\}_{n=0}^{\infty}$ в (18) такова, что выполнено условие (20).

Решение интерполячионной задачи $A-\Gamma(18),(19)$ в пространстве $A(\exp , D)$ существует тогда и только тогда, когда сущ,стввет пара функиий $\left\{f_{j}(w)\right\}_{j=0}^{1}$ $\left(f_{0}(w)\right.$ - четная, $f_{1}(w)$ - нечетная $)$, удовлетворяющая условию

$$
\left\{g_{0}(W(z))+f_{0}(W(z))\right\} e^{-b_{0} z}-\left\{g_{1}(W(z))+f_{1}(W(z))\right\} e^{-b_{1} z} \in A(D),
$$

əде $w=z e^{z}$. 
ДокАЗАТЕЛЬСтво. Необходимость. Пусть интерполяционная задача А-Г (18), (19) разрешима в пространстве $A(\exp , D)$. В таком случае существует функция $F(z) \in$ $A(\exp , D)$, удовлетворяющая $(18)$, с узлами интерполяции $(19)$. Используя $(1)$, получим

$$
\frac{1}{2 \pi i} \int_{\Gamma_{t}} t^{n} e^{n t} e^{b(n) t} \gamma(t) d t=a_{n}, \quad n=0,1,2, \ldots,
$$

здесь $\gamma(t) \not \equiv 0, \gamma(z) \in A_{0}\left(\overline{\mathbb{C}} \backslash \operatorname{int} \Gamma_{t}\right), \Gamma_{t}$ - жорданов спрямляемый кусочно-гладкий путь, достаточно близкий к гранище области $D, \Gamma_{t} \subset D$. Из (22) вытекает

$$
\left\{\begin{array}{l}
\frac{1}{2 \pi i} \int_{\Gamma_{t}} t^{2 n} e^{2 n t} e^{b_{0} t} \gamma(t) d t=a_{2 n}, \\
\frac{1}{2 \pi i} \int_{\Gamma_{t}} t^{2 n+1} e^{(2 n+1) t} e^{b_{1} t} \gamma(t) d t=a_{2 n+1}, \quad n=0,1,2, \ldots
\end{array}\right.
$$

Таким образом, получаем М-проблему моментов при $p=2, W_{0}(z) \equiv W_{1}(z)=z e^{z}$, $A_{0}(z)=e^{b_{0} z}, A_{1}(z)=e^{b_{1} z}$. Из теоремы 1 и (20) заключаем, что существует пара функций $f_{j}(w) \in A_{0}(C G), j=0,1, f_{0}(w)$ четная, $f_{1}(w)$ нечетная, удовлетворяющие условию

$$
\left\{g_{0}\left(W_{0}(z)\right)+f_{0}\left(W_{0}(z)\right)\right\} W_{0}^{\prime}(z) e^{b_{1} z}-\left\{g_{1}\left(W_{1}(z)\right)+f_{1}\left(W_{1}(z)\right)\right\} W_{1}^{\prime}(z) e^{b_{0} z} \in A(D) .
$$

Учитьвая, что $W_{0}(z) \equiv W_{1}(z)$ и $W_{0}^{\prime}(z) \neq 0$ в $D$, получаем $(21)$.

Достаточность. Пусть вьполнено условие (20) и существует пара функций $f_{j}(w)$, $j=0,1$, удовлетворяющая (21). В таком случае имеет место (24). Применяя теорему 1 (при $p=2$ ), заключаем, что существует функция $\Phi_{0}^{-}(z) \not \equiv 0, \Phi_{0}^{-}(z) \in A_{0}(C D)$, для которой справедливы соотношения

$$
\left\{\begin{array}{l}
\frac{1}{2 \pi i} \int_{\Gamma_{t}}\left[W_{0}(t)\right]^{2 n} e^{b_{0} t} \Phi_{0}^{-}(t) d t=a_{2 n}, \\
\frac{1}{2 \pi i} \int_{\Gamma_{t}}\left[W_{1}(t)\right]^{2 n+1} e^{b_{1} t} \Phi_{0}^{-}(t) d t=a_{2 n+1},
\end{array} \quad n=0,1,2, \ldots\right.
$$

Отсюда заключаем, что задача $\mathrm{A}-\Gamma(18),(19)$ имеет решение $F(z)$ в пространстве $A(\exp , D)$ :

$$
F(z)=\frac{1}{2 \pi i} \int_{\Gamma_{t}} e^{z t} \Phi_{0}^{-}(t) d t
$$

Теорема доказана.

ЗАмечание 6 . Теорема 2 сводит задачу А-Г $(18),(19)$ к поиску пары функций $f_{j}(w)$ $\not \equiv 0, j=0,1, f_{0}(w)$ четная, а $f_{1}(w)$ нечетная, $f_{j}(w) \in A_{0}(C G)$, удовлетворяющих условию:

$$
\left\{g_{0}(w)+f_{0}(w)\right\} e^{-b_{0} \psi(w)}-\left\{g_{1}(w)+f_{1}(w)\right\} e^{-b_{1} \psi(w)} \in A(G),
$$

где $g_{j}(w), j=0,1,-$ функции, описанные в $(20), z=\psi(w)$ - функция, обратная $\mathrm{k}$ $w=z e^{z}, G=W(D)$.

ЗАмЕчАниЕ 7. Теорема 2 дает вид решения интерполяционной задачи $\mathrm{A}-\Gamma(18),(19)$, т.е. решает проблему конструкции в упомянутой выше интерполящионной задаче. 
ЗАмЕчАнИЕ 8. Если положить, что $b_{0}=\tau, b_{1}=-\tau, \tau \in \mathbb{C}$, то последовательность узлов интерполящии (19) превращается в последовательность

$$
\lambda_{n}=n+(-1)^{n} \tau, \quad \tau \in \mathbb{C},
$$

изученную ранее в других работах (см. [10]-[12]).

ЗАмЕчАниЕ 9 . В теореме 2 область $G=W(D)\left(w=z e^{z}\right)$ не обязана быть симметричной относительно начала координат, как это требовалось бы при применении метода Казьмина (см. [3]).

2.2. Нахождение пространств единственности задачи Абеля-Гончарова. Обозначим через $\Delta\left(w ; b_{0}, b_{1}\right)$ следующую функцию:

$$
\Delta\left(w ; b_{0}, b_{1}\right)=(-1)\left\{\exp \left[b_{0} \psi(w)+b_{1} \psi(-w)\right]+\exp \left[b_{0} \psi(-w)+b_{1} \psi(w)\right]\right\}
$$

Легко проверить, что $\Delta\left(w ; b_{0}, b_{1}\right)$ имеет нули только в точках корней уравнений

$$
\psi(w)-\psi(-w)= \pm \frac{\pi i}{b_{0}-b_{1}}
$$

Возможны следующие случаи:

a) $b_{0}-b_{1} \in \mathbb{R}, b_{0}-b_{1} \neq 0$;

b) $b_{0}-b_{1} \in \mathbb{C}, b_{0}-b_{1} \neq 0$.

Рассмотрим их по отдельности.

а) Пусть $b_{0}-b_{1} \in \mathbb{R}, b_{0}-b_{1} \neq 0$. Обозначим через $2 \tau=b_{0}-b_{1}$, тогда $\tau \neq 0$ и

$$
\psi(w)-\psi(-w)= \pm \frac{\pi i}{2 \tau}
$$

Выбирая знак в правой части совпадающим со знаком $\tau$, получим

$$
\psi(w)-\psi(-w)= \pm \frac{\pi i}{|2 \tau|}, \quad \tau \in \mathbb{R}, \quad \tau \neq 0 .
$$

Корни уравнения (28) принадлежат положительной части мнимой оси в $\mathbb{C}_{w}(\mathrm{cm.}$. 11$\left.]\right)$, пусть $w^{*}-$ ближайший к началу координат корень уравнения (28). Обозначим через $p^{+}$ разрез в $\mathbb{C}_{w}$ следующего вида: он начинается в точке $w^{*}$ и продолжается вдоль положительной части мнимой оси в бесконечность; $p^{-}-$разрез в $\mathbb{C}_{w}$, симметричньй с $p^{+}$ относительно начала координат. Таким образом, в $\mathbb{C}_{w} \backslash\left\{p^{+} \cup p^{-}\right\}$нет нулей функции $\Delta\left(w ; b_{0}, b_{1}\right) \quad\left(b_{0}-b_{1} \in \mathbb{R}\right)$. Образы разрезов $p^{+}$и $p^{-}$при отображении $z=\psi(w)$, обратном к $w=z e^{z}$, обозначим через $\gamma^{+}$и $\gamma^{-}$соответственно. Кривые $\gamma^{+}$и $\gamma^{-}$симметричны друг другу относительно действительной оси, они принадлежат области $U$ и расположены в верхней и нижней полуплоскости $\mathbb{C}_{z}$ соответственно.

Можно описать $\gamma^{+}$более подробно (см. [12]). Кривая $\gamma^{+}$начинается в точке

$$
z^{*}=x^{*}+i y^{*}, \quad x^{*}=\frac{\pi}{4|\tau|} \operatorname{tg} \frac{\pi}{4|\tau|}, \quad y^{*}=\frac{\pi}{4|\tau|}, \quad z^{*}=\psi\left(w^{*}\right), \quad 2 \tau=b_{0}-b_{1},
$$

и продолжается вдоль кривой, заданной уравнением $x=y \operatorname{tg} y, y \in[\pi /(4|\tau|) ; \pi / 2)$, в бесконечность. 
ТЕОремА 3. Пусть $D$ - ограниченная односвязная область с односвязным дополнением $C D$ до $\overline{\mathbf{C}}_{z}, \bar{D} \subset U \backslash\left\{\gamma^{+} \cup \gamma^{-}\right\} u G=W(D)\left(w=z e^{z}\right)$.

Если числовая последовательность $\left\{a_{n}\right\}_{n=0}^{\infty}$ в (18) удовлетворяет условиям (20), то в пространстве $A_{0}(C G)$ существует единственная пара функиий $f_{j}(w)$, $j=0,1\left(f_{0}(w)\right.$ четная, а $f_{1}(w)$ нечетная), удовлетворяющая условию (21) при $b_{0}-b_{1} \in \mathbb{R}$.

ДокАЗАТЕЛЬСтво. Рассмотрим следующую краевую задачу: найти функции $\Phi_{j}^{-}(w) \in A_{0}(C G), \Phi_{j}^{-}(w) \not \equiv 0, j=0,1$, и $\Phi^{+}(w) \in A(G)$, удовлетворяюшие следующему краевому условию на контуре $\Gamma_{w} \subset G\left(\Gamma_{w}\right.$ достаточно близок к границе области $\left.G\right)$ :

$$
\left\{g_{0}(w)+\Phi_{0}^{-}(w)\right\} e^{-b_{0} \psi(w)}-\left\{g_{1}(w)+\Phi_{1}^{-}(w)\right\} e^{-b_{1} \psi(w)}=\Phi^{+}(w) .
$$

В случае, если мы найдем четную функцию $\Phi_{0}^{-}(w)$ и нечетную функцию $\Phi_{1}^{-}(w)$, удовлетворяющие (29), то утверждение теоремы будет доказано.

В силу (20) из (29) получаем систему краевых задач на контуре $\Gamma_{w}$ :

$$
\left\{\begin{array}{c}
\Phi_{0}^{-}(w) e^{-b_{0} \psi(w)}-\Phi_{1}^{-}(w) e^{-b_{1} \psi(w)} \\
=\Phi^{+}(w)+g_{1}(w) e^{-b_{1} \psi(w)}-g_{0}(w) e^{-b_{0} \psi(w)} \\
\Phi_{0}^{-}(w) e^{-b_{0} \psi(-w)}+\Phi_{1}^{-}(w) e^{-b_{1} \psi(-w)} \\
=\Phi^{+}(w)+g_{1}(w) e^{-b_{1} \psi(-w)}+g_{0}(w) e^{-b_{0} \psi(-w)}
\end{array}\right.
$$

Отсюда следует система краевых условий (задач) на контуре $\Gamma_{w}$ :

где

$$
\left\{\begin{array}{l}
\Phi_{0}^{-}(w)=\frac{1}{\beta(w)}\left\{\widehat{\Phi}_{0}^{+}(w)+g_{0}^{*}(w)\right\}, \\
\Phi_{1}^{-}(w)=\frac{1}{\beta(w)}\left\{\widehat{\Phi}_{1}^{+}(w)+g_{1}^{*}(w)\right\}
\end{array}\right.
$$

$$
\beta(w)=-\frac{\Delta\left(w ; b_{0}, b_{1}\right)}{\exp \left\{(\psi(w)+\psi(-w))\left(b_{0}+b_{1}\right)\right\}},
$$

a функции $g_{j}^{*}(w), j=0,1$, имеют следуюший вид:

$$
\begin{aligned}
g_{0}^{*}(w)= & \frac{2 g_{1}(w)}{\exp \left\{b_{1}[\psi(w)+\psi(-w)]\right\}} \\
& +\frac{g_{0}(w)}{\exp \left\{b_{0} \psi(-w)+b_{1} \psi(w)\right\}-\exp \left\{b_{0} \psi(w)+b_{1} \psi(-w)\right\}}, \\
g_{1}^{*}(w)= & g_{1}(w)\left\{\frac{1}{\exp \left\{b_{0} \psi(w)+b_{1} \psi(-w)\right\}}-\frac{1}{\exp \left\{b_{0} \psi(-w)+b_{1} \psi(w)\right\}}\right\} \\
& +\frac{2 g_{0}(w)}{\exp \left\{b_{0}[\psi(w)+\psi(-w)]\right\}} .
\end{aligned}
$$

Нетрудно проверить, что $g_{0}^{*}(w)$ четная, а $g_{0}^{*}(w)$ нечетная функции. Число решений и разрешимость каждой из краевых задач в (30) зависит от индекса

$$
\chi=\operatorname{ind}_{\Gamma_{w}}(\beta(w)),
$$

который в нашем случае равен числу нулей функции $\Delta\left(w ; b_{0}, b_{1}\right)$, попавших внутрь контура $\Gamma_{w}$ (см. [5]). В свою очередь, нули $\Delta\left(w ; b_{0}, b_{1}\right)$ зависят от $b_{0}, b_{1}$. В нашем случае $b_{0}-b_{1} \in \mathbb{R}$, поэтому в области $G$ нет нулей $\Delta\left(w ; b_{0}, b_{1}\right)$ и, следовательно, $\chi=0$. В этом случае существует единственное и отличное от нуля решение $\Phi_{0}^{-}$и $\Phi_{1}^{-}$каждой из краевых задач (30) (см. [5]), причем $\Phi_{0}^{-}$четная, а $\Phi_{1}^{-}$нечетная функции (последнее следует из вида функций $g_{0}^{*}(w)$ и $\left.g_{1}^{*}(w)\right)$. Теорема доказана. 
ЗАмечАниЕ 10 . В условиях теоремы 3 область $D$ такова, что вьполнено $\bar{D} \subset U \backslash$ $\left\{\gamma^{+} \cup \gamma^{-}\right\}$. Фактически же достаточно потребовать только, чтобы $\bar{D} \subset U$ и в области $G=W(D)\left(w=z e^{z}\right)$ не было нулей функции $\Delta\left(w ; b_{0}, b_{1}\right)$.

ТЕОремА 4. Пусть $D$ - ограниченная односвязная область с односвязным дополнением $C D$ до $\overline{\mathbb{C}}_{z}, \bar{D} \subset U \backslash\left\{\gamma^{+} \cup \gamma^{-}\right\}$. Если числовая последовательность $\left\{a_{n}\right\}_{n=0}^{\infty}$ в (18) удовлетворяет условию (20) и $b_{0}-b_{1} \in \mathbb{R}$, то решение интерполяиионной задачи $A-\Gamma(18)-(19)$ существует и является единственным в пространстве $A(\exp , D)$.

ДокАЗАТЕЛЬСТво. Следует из теоремы 2 , замечания 6 и теоремы 3.

СлЕДСТВИЕ 1. Пусть $D$ - ограниченная односвязная область с односвязным дополнением $C D$ до $\overline{\mathbb{C}}_{z}, \bar{D} \subset U$. Если числовая последовательность $\left\{a_{n}\right\}_{n=0}^{\infty}$ в (18) удовлетворяет условию (20), то решение интерполячионной задачи $A-\Gamma(18)-(19)$ при $b_{0}-b_{1} \in \mathbb{R}$ существует, но оно ужсе не является единственным в пространстве $A(\exp , D)$.

ДоКАЗАТЕЛЬСТво следует из того, что в области $G=W(D)\left(w=z e^{z}\right)$ появляются нули функции $\Delta\left(w ; b_{0}, b_{1}\right)$. В этом случае краевые задачи $(30)$ имеют уже не единственные решения $\Phi_{0}^{-}(w)$ и $\Phi_{1}^{-}(w)$, удовлетворяющие $(29)$. Используя теорему 2 и замечание 6 , получаем требуемое.

b) $b_{0}-b_{1} \in \mathbb{C}, b_{0}-b_{1} \neq 0$.

Этот случай рассматривается полностью аналогично пункту а). Получаются результаты, дословно повторяющие утверждения теорем 3 и 4 (с небольшими вариациями).

2.3. Полнота некоторых систем аналитических функций. Пусть числовая совокупность $\bigcup_{j=0}^{p-1}\left\{a_{n j}\right\}_{n=0}^{\infty}$ в (3) есть совокупность нулей.

Спрашивается, существует ли функция $g(z)$ такая, что $g(z) \in A_{0}(C D), g(z) \not \equiv 0$, для которой имеют место равенства

$$
\begin{gathered}
\frac{1}{2 \pi i} \oint_{\Gamma_{z}}\left[W_{j}(z)\right]^{n p+l_{j}} A_{j}(z) g(z) d z=0, \\
j=0,1,2, \ldots, p-1, \quad 0 \leqslant l_{j} \leqslant p-1, \quad l_{j} \in \mathbb{N} \cup\{0\}, \quad n=0,1,2, \ldots .
\end{gathered}
$$

Задача поиска такой функции $g(z)$ эквивалентна неполноте системы функций

$$
\begin{gathered}
\bigcup_{j=0}^{p-1}\left[W_{j}(z)\right]^{n p+l_{j}} A_{j}(z), \\
j=0,1,2, \ldots, p-1, \quad 0 \leqslant l_{j} \leqslant p-1, \quad l_{j} \in \mathbb{N} \cup\{0\}, \quad n=0,1,2, \ldots,
\end{gathered}
$$

в пространстве $A(D)$ (см. [2], [3]). Из теоремы 1 следует

ТЕОРемА 5. Пусть $D$ - любая односвязная ограниченная область с односвязным дополнением $C D$ до $\overline{\mathbb{C}}_{z}$.

Система функиий

$$
\begin{gathered}
\bigcup_{j=0}^{p-1}\left[W_{j}(z)\right]^{n p+l_{j}} A_{j}(z), \\
j=0,1,2, \ldots, p-1, \quad 0 \leqslant l_{j} \leqslant p-1, \quad l_{j} \in \mathbb{N} \cup\{0\}, \quad p \in \mathbb{N}, \quad p \geqslant 2, \quad A_{j}(z) \neq 0,
\end{gathered}
$$


полна в пространстве $A(D)$ тогда и только тогда, когда не существует ни одной совокупности из р функиий $\left\{f_{j}(w)\right\}_{j=0}^{p-1}, p \in \mathbb{N}, p \geqslant 2$, обладающих следующ,ими свойствами:

1) $f_{j}(w) \in A_{0}\left(C G_{j}\right), f_{j}(w) \not \equiv 0, j=0,1,2, \ldots, p-1$;

2) лорановские части функиий $f_{j}(w)$ не имеют степеней вида $1 / w^{n p+l_{j}+1}$;

3) система функиий $\left\{f_{j}(w)\right\}_{j=0}^{p-1}$ удовлетворяет условию

$$
f_{0}\left(W_{0}(z)\right) W_{0}^{\prime}(z) A_{j}(z)-f_{j}\left(W_{j}(z)\right) W_{j}^{\prime}(z) A_{0}(z) \in A(D), \quad j=1,2, \ldots, p-1 .
$$

Рассмотрим применение теоремы 5 (при $p=2$ ) к вопросу полноты некоторых систем аналитических функций в пространстве $A(D)$.

Пусть $D_{R}$ - круг радиуса $R: D_{R}=\{z:|z|<R, R>0\}$, а функции $W_{0}(z)$ и $W_{1}(z)$ являются голоморфными, однолистными и нечетными в $D_{R}$. Кроме того, рассмотрим функции $A_{0}(z), A_{1}(z)$, которые являются голоморфными в $D_{R}$ и не имеют там нулей. Через $A_{R}$ обозначим пространство $A\left(D_{R}\right)$. Справедлива

Теорема 6. Система функиий

$$
\left\{\left[W_{0}(z)\right]^{2 n} A_{0}(z)\right\}_{n=0}^{\infty} \cup\left\{\left[W_{1}(z)\right]^{2 n+1} A_{1}(z)\right\}_{n=0}^{\infty}
$$

полна в пространстве $A_{R}$ тогда и только тогда, когда функиия

$$
\varphi(z) \equiv A_{0}(z) A_{1}(-z)+A_{0}(-z) A_{1}(z)
$$

не имеет нулей в области $D_{R}$.

\section{СПИСОК ЦИТИРОВАННОЙ ЛИТЕРАТУРЫ}

[1] Евграфов М. А. Основные понятия интерполяции целых функций. М.: Препринт ИПМ $\mathrm{PAH}, 1975$.

[2] Ибрагимов И. И. Методы интерполяции и некоторые их применения. М.: Наука, 1971.

[3] Казьмин Ю.А. Общая проблема моментов в комплексной области // Proceedings of the Conference on Constructive Theory of Functions (Budapest, 1969). Budapest, 1972. P. 225-254.

[4] Казьмин Ю.А. К проблеме моментов в комплексной области // Докл. АН СССР. 1972. T. 204. №6. C. 1309-1312.

[5] Гахов Ф. Д. Краевые задачи. М.: Наука, 1977.

[6] Евграфов М. А. Интерполяционная задача Абеля-Гончарова. М.: Гостехиздат, 1954.

[7] Гончаров В.Л. Интерполяционные процессы и целые функции // УМН. 1937. Т. 3. C. $113-143$.

[8] Гельфонд А.О. Проблема представления и единственности аналитической функции первого порядка // УМН. 1937. Т. 3. С. 144-174.

[9] Гельфонд А. О. Исчисление конечных разностей. М.: Наука, 1967.

[10] Осколков В. А. Об одном классе узлов, для которых справедлива точная оценка интерполяционных полиномов Гончарова // Тр. МИХМ. 1973. Т. 50. С. 34-38.

[11] Адибекян Р. В. Об интерполяционной задаче Абеля-Гончарова для целых функций экспоненциального типа // Уч. зап. Ереванского гос. ун-та. Сер. естеств. науки. 1983. Т. 3. C. $20-27$.

[12] Андриянов Г. И. Интерполяционная задача Абеля-Гончарова. Дисс. . . к. ф.-м...М., 1998. 